\title{
Binary Evolutionary Models
}

\author{
Z. Han $^{1} \&$ Ph. Podsiadlowski ${ }^{2}$ \\ ${ }^{1}$ National Astronomical Observatories/Yunnan Observatory, Kunming, 65011, P.R. China \\ email: zhanwenhan@hotmail.com \\ ${ }^{2}$ Department of Physics, University of Oxford, Keble Road, Oxford OX1 3RH, UK \\ email: podsi@astro.ox.ac.uk
}

\begin{abstract}
In this talk, we present the general principles of binary evolution and give two examples. The first example is the formation of subdwarf B stars (sdBs) and their application to the long-standing problem of ultraviolet excess (also known as UV-upturn) in elliptical galaxies. The second is for the progenitors of type Ia supernovae (SNe Ia). We discuss the main binary interactions, i.e., stable Roche lobe overflow (RLOF) and common envelope (CE) evolution, and show evolutionary channels leading to the formation of various binary-related objects. In the first example, we show that the binary model of sdB stars of Han et al. (2002, 2003) can reproduce field sdB stars and their counterparts, extreme horizontal branch (EHB) stars, in globular clusters. By applying the binary model to the study of evolutionary population synthesis, we have obtained an "a priori" model for the UV-upturn of elliptical galaxies and showed that the UV-upturn is most likely resulted from binary interactions. This has major implications for understanding the evolution of the UV excess and elliptical galaxies in general. In the second example, we introduce the single degenerate channel and the double degenerate channel for the progenitors of SNe Ia. We give the birth rates and delay time distributions for each channel and the distributions of companion stars at the moment of SN explosion for the single degenerate channel, which would help to search for the remnant companion stars observationally.
\end{abstract}

Keywords. binaries: close, galaxies: elliptical and lenticular, cD, stars: evolution, subdwarfs, supernovae: general, white dwarfs, ultraviolet: galaxies

\section{General Principles of Binary Evolution}

About half of the stars are in binaries and binary evolution plays a crucial role in the formation of many interesting objects, such as Algols, FK Comae (FK Com) stars, cataclysmic variables $(\mathrm{CVs})$, planetary nebulae $(\mathrm{PNe})$, barium $(\mathrm{Ba})$ stars, $\mathrm{CH}$ stars, type Ia supernovae (SNe Ia), AM Canum Venaticorum (AM CVn) stars, low mass Xray binaries (LMXB), high mass X-ray binaries (HMXB), symbiotic (Sym) stars, blue stragglers (BSs), pulsars, subdwarf B (sdB) stars, double degenerates (DDs) etc. Binary evolution is also important in the study of evolutionary population synthesis, which is a power tool in the study of galaxies.

A binary system (of low/intermediate mass) has two components: the primary (the initially more massive one) and the secondary. As the binary evolves, the primary expands and may fill its Roche lobe on the Hertzsprung gap or on the giant branch. Roche lobe overflow (RLOF) begins and the primary's envelope mass transfers to the secondary. Given the mass ratio $q$ of primary to secondary less than a critical value $q_{\mathrm{c}}$ (Hjellming \& Webbink, 1987; Webbink, 1988; Han \& Webbink, 1999; Han et al., 2002) at the onset of the mass transfer, where $q_{\mathrm{c}}$ mainly depends on the entropy profile of the primary's envelope and the angular momentum loss from the system, the mass transfer is stable, leading to a wide white dwarf (WD) binary. Given the mass ratio $q$ larger than $q_{\mathrm{c}}$, the mass transfer is dynamically unstable, leading to the formation of a common envelope 


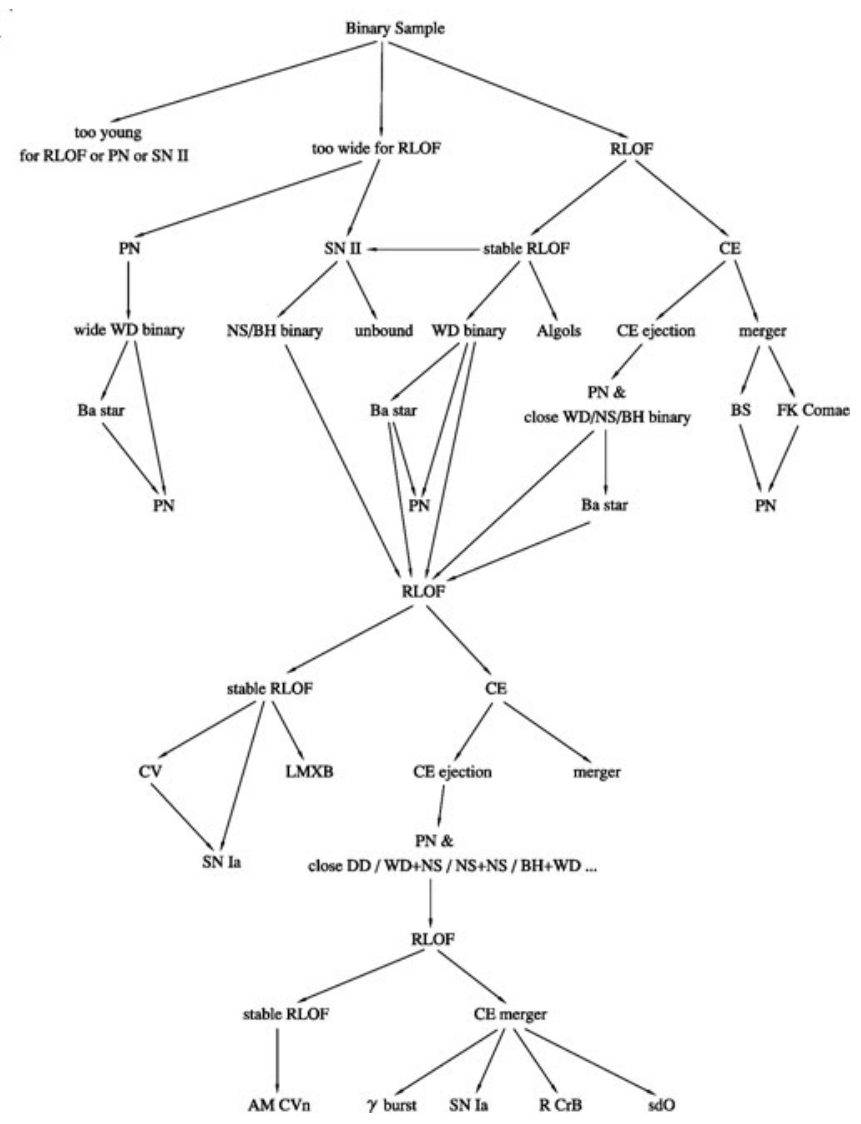

Figure 1. A simplified version of flow chart of binary evolution. See paragraph 1 of Section 1 of the text for the acronyms.

(CE; Paczyński, 1976). The CE engulfs the core of the primary and the secondary, and does not co-rotate with the embedded binary. The friction between the $\mathrm{CE}$ and the embedded binary makes the orbit decay, and a large amount of orbital energy released is deposited into the CE. If the CE can be ejected, a close WD binary forms, otherwise a fast rotating merger is resulted. For a WD binary system, the secondary continues to evolve and may experience mass transfer. Similar to the process described above, the mass transfer may lead to the formation of a $\mathrm{CE}$ and the $\mathrm{CE}$ ejection produces a double degenerate.

Figure 1 is a flow chart of binary evolution. It is by no means comprehensive, but it does show evolutionary channels leading to various objects for a binary system with given conditions.

A good stellar evolution theory should give and predict the statistical properties of a stellar population as well as the properties of individual stars or binaries. Binary population synthesis (BPS) is to evolve a large number of stars (including binaries) in order to investigate statistical properties of stars and check evolutionary mechanisms for different types of stars. In a BPS study, we first generate a binary sample (10 million binaries), then evolve the sample according to stellar evolution model grids and taking into account binary interactions, and we obtain different types of binary-related objects, which can be directly compared to observations. 
In addition to the grids of stellar evolutionary models, we adopt the following input in BPS simulations:

(1) A constant star-formation rate is taken over the last 15 Gyr for a field population, or alternatively, a single star burst for a globular cluster or an elliptical galaxy.

(2) The initial mass function of Miller \& Scalo (1979) is adopted.

(3) We mainly adopt a constant initial mass ratio distribution $n\left(q^{\prime}\right)$, where $q^{\prime}=1 / q$ is the ratio of secondary to primary.

(4) We take the distribution of separations to be constant in $\log a$ for wide binaries, where $a$ is the orbital separation. The adopted distribution gives that $\sim 50 \%$ of stellar systems are binary systems with orbital periods less than $100 \mathrm{yr}$.

The main model parameters in BPS are mass transfer efficiency $\alpha_{\mathrm{RLOF}}$ for the first stable RLOF (where the accretor is a main sequence star), CE ejection efficiency $\alpha_{\mathrm{CE}}$ and thermal contribution $\alpha_{\mathrm{th}}$ for CE evolution. The mass transfer efficiency $\alpha_{\mathrm{RLOF}}$ is the fraction of the envelope mass that is transferred onto the secondary rather than is ejected from the system, where we assume that the matter lost from the system carries away the specific angular momentum of the system. A typical value for $\alpha_{\mathrm{RLOF}}$ is 0.5 . The $\mathrm{CE}$ ejection efficiency $\alpha_{\mathrm{CE}}$ is the fraction of the released orbital energy used to overcome the binding energy of the envelope during the spiral-in process of a CE. The thermal contribution $\alpha_{\mathrm{th}}$ defines the fraction of the internal energy of thermodynamics (including recombination energy as well as the thermal energy) contributing to the binding energy of the CE. A CE is ejected if

$$
\alpha_{\mathrm{CE}} \Delta E_{\mathrm{orb}} \geqslant E_{\mathrm{gr}}-\alpha_{\mathrm{th}} E_{\mathrm{th}}
$$

where $\Delta E_{\text {orb }}$ is the orbital energy released during the spiral-in process, $E_{\text {gr }}$ the gravitational binding energy of the CE, $E_{\mathrm{th}}$ the internal energy of the CE. Both $E_{\mathrm{gr}}$ and $E_{\mathrm{th}}$ are calculated from detailed stellar models and therefore the prescription here is different from the $\lambda$ prescription but appear to be more physical. We refer the reader to Han, Podsiadlowski \& Eggleton (1994), Dewi \& Tauris (2000) and Podsiadlowski, Rappaport \& Han (2003) for the details. The inclusion of the internal energy in ejecting the CE seems to be the most plausible in the explanation of both long-period and short-period binaries containing compact objects (Han et al., 1995a; 1995b; Dewi \& Tauris, 2000). Webbink (2007) has made a physical investigation on both the energetics of CE evolution and the angular momentum prescription (i.e. the $\gamma$ prescription, Nelemans et al., 2000), and convincingly showed the necessity of recombination energy term for common envelope evolution. Previous studies, e.g., Han, Eggleton, Podsiadlowski \& Tout (1995), Han, Podsiadlowski \& Eggleton (1995), Han (1998), Han et al. (2002), Han et al. (2003), Han \& Podsiadlowski (2004), have showed that both $\alpha_{\mathrm{th}}$ and $\alpha_{\mathrm{th}}$ are close to one.

\section{The binary model for subdwarf B stars and the UV-upturn of elliptical galaxies}

Subdwarf B (sdB) stars $\dagger$ are core helium-burning stars with very thin hydrogen envelope (Heber, 1986). They are important in many aspects of astrophysics, e.g., stellar evolution, distance indicators, Galactic structure, and the long-standing problem of farultraviolet excess in early-type galaxies (Kilkenny et al., 1997; Green, Schmidt \& Liebert, 1986; Han, Podsiadlowski \& Lynas-Gray, 2007).

$\dagger$ In this paper, we collectively refer to helium-core-burning stars with thin hydrogen envelopes as sdB stars, even if some of them may in reality be sdO or sdOB stars 
Maxted et al. (2001) showed that the majority of field sdB stars are in binaries, and this has posed a serious challenge to stellar evolution theory. Han et al. $(2002 ; 2003)$ proposed a binary model for their formation. In the model, there are three types of formation channels for sdB stars: stable RLOF for sdB binaries with long orbital periods, $\mathrm{CE}$ ejection for sdB binaries with short orbital periods, and the merger of helium WDs to form single EHB stars. In the stable RLOF channel, the mass donor fills its Roche lobe near the tip of the first giant branch and experiences a stable mass transfer, and its envelope is striped off by the RLOF, and the naked helium core (with thin hydrogen envelope) get ignited to produce a sdB stars. In the CE ejection channel, the mass donor also fills its Roche lobe near the tip of the first giant branch to have a dynamically unstable mass transfer leading to the formation of a CE. The CE ejection leaves a naked helium core (with thin hydrogen envelope) and the naked helium core is ignited to produce a sdB star. In the CE channel, the donor star needs to fill its Roche lobe closer to the tip of the first giant branch, or, in other words, the minimum core mass required for the donor star at the onset of mass transfer is larger than that in the stable RLOF channel to produce a sdB star. This is simply because that the time scale of the CE evolution is much shorter than that of the stable RLOF and the core does not grow by much in the CE process. In the merger channel, a close helium WD pair coalesces due to angular momentum loss via gravitational wave radiation. The binary model of Han et al. (2002; 2003) has successfully explained the main observational characteristics of field sdB stars: their distributions in the orbital period-minimum companion mass diagram, and in the effective temperature-surface gravity diagram; their distributions of orbital period and mass function; their binary fraction and the fraction of sdB binaries with WD companions; their birth rates; and their space density. The model is indeed a step forward and is widely used in the study of sdB stars (O'Tool, Heber \& Benjamin, 2004).

Moni Bidin et al. (2006), Moni Bidin, Catelan \& Altmann (2008) have done radialvelocity surveys for extreme horizontal branch (EHB) stars, the counterparts of field sdB stars, in globular clusters. They found that there is a remarkable lack of close binary systems in EHB stars. This is surprising as compared to the high binary fraction in field sdB stars. They speculated that there may exist a binary fraction-age relation for sdB stars. Han (2008) showed that such a relation does exist and the binary model of Han et al. $(2002 ; 2003)$ can reproduce the EHB stars in globular clusters, in particular, the low binary fraction of the EHB stars. The main reason for the low binary fraction is that the stars in a globular cluster are all old, and the envelopes of donor stars in the CE channel are loosely bound, leading to wide EHB binaries rather than close ones.

One of the first major discoveries soon after the advent of UV astronomy was the discovery of an excess of light in the far-ultraviolet (far-UV) in elliptical galaxies (see the review by O'Connell, 1999). This came as a complete surprise since elliptical galaxies were supposed to be entirely composed of old, red stars and not to contain any young stars that radiate in the UV. Since then it has become clear that the far-UV excess (or upturn) is not a sign of active contemporary star formation, but is caused by an older population of helium-burning stars or their descendants with a characteristic surface temperature of 25,000 K (Ferguson et al., 1991).

The origin of this population of hot, blue stars in an otherwise red population has, however, remained a major mystery. As we described above, the binary model of Han et al. $(2002 ; 2003)$ reproduces Galactic hot subdwarfs (synonymous with sdB stars in this paper). The key feature of the channels in the model is that they provide the missing physical mechanism for ejecting the envelope and for producing a hot subdwarf. Moreover, since it is known that these hot subdwarfs provide an important source of far-UV light in our own Galaxy, it is not only reasonable to assume that they will also 


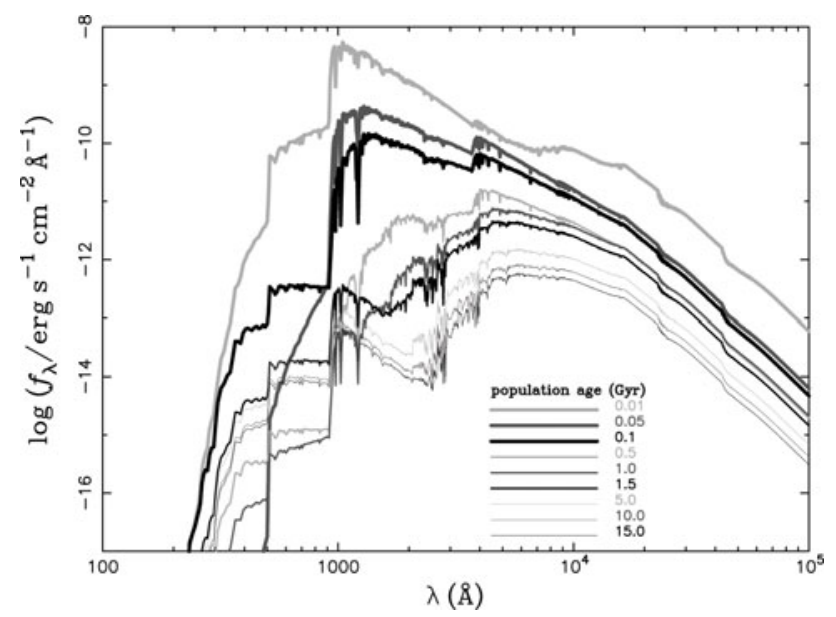

Figure 2. The evolution of the rest-frame intrinsic spectral energy distribution (SED) for a simulated galaxy in which all stars formed at the same time, i.e. a simple stellar population (SSP). The stellar population (including binaries) has a mass of $10^{11} M_{\odot}$ and the galaxy is assumed to be at a distance of $10 \mathrm{Mpc}$. The figure is for the standard simulation set (with $\alpha_{\mathrm{CE}}=\alpha_{\mathrm{th}}=0.75$ and $\left.\alpha_{\mathrm{RLOF}}=0.5\right)$ in Han, Podsiadlowski \& Lynas-Gray (2007) and no offset is applied to the SEDs. Note that the line sections of between $500 \AA$ and $900 \AA$ for population ages of 1.5 and 5.0 Gyr overlap.

contribute significantly to the far-UV in elliptical galaxies, but is in fact expected. It would, therefore, be "a priori" to apply the Han et al. model to the study of the UVupturn problem.

To quantify the importance of the effects of binary interactions on the spectral appearance of elliptical galaxies, we have performed the first population synthesis study of galaxies that includes binary evolution (see also Bruzual \& Charlot, 1994; Worthy, 1994; Zhang, Li \& Han, 2005). It is based on a binary population synthesis model of Han et al. $(2002 ; 2003)$ that has been calibrated to reproduce the short-period hot subdwarf binaries in our own Galaxy that make up the majority of Galactic hot subdwarfs (Maxted et al., 2001). The population synthesis model follows the detailed time evolution of both single and binary stars, including all binary interactions, and is capable of simulating galaxies of arbitrary complexity, provided that the star-formation history is specified. To obtain galaxy colours and spectra, we have calculated detailed grids of spectra for hot subdwarfs using the ATLAS9 (Kurucz, 1992) stellar atmosphere code, which calculates plane-parallel atmospheres in local thermodynamic equilibrium. For the spectra and colours of single stars with hydrogen-rich envelopes, we use the comprehensive BaSeL library of theoretical stellar spectra (Lejeune, Cuisinier \& Buser, 1997; 1998).

Figure 2 shows our simulated evolution of the spectral energy distribution (SED) of a galaxy in which all the stars formed at the same time. The total mass of the stellar population (including binaries) is $10^{11} M_{\odot}$ and the galaxy is taken to be at a distance of $10 \mathrm{Mpc}$. At early times, the far-UV flux is entirely caused by the contribution from young stars. Hot subdwarfs from the various binary evolution channels become important after about $1.1 \mathrm{Gyr}$, which corresponds to the evolutionary timescale of a $2 M_{\odot}$ star, and soon start to dominate completely. After a few Gyr the far-UV SED no longer changes appreciably relative to the visual flux. One immediate implication of this is that the model predicts that the magnitude of the UV excess $(1550-V)$, defined as the relative ratio of the flux in the $V$ band to the far-UV flux (Burstein et al., 1988), should not 


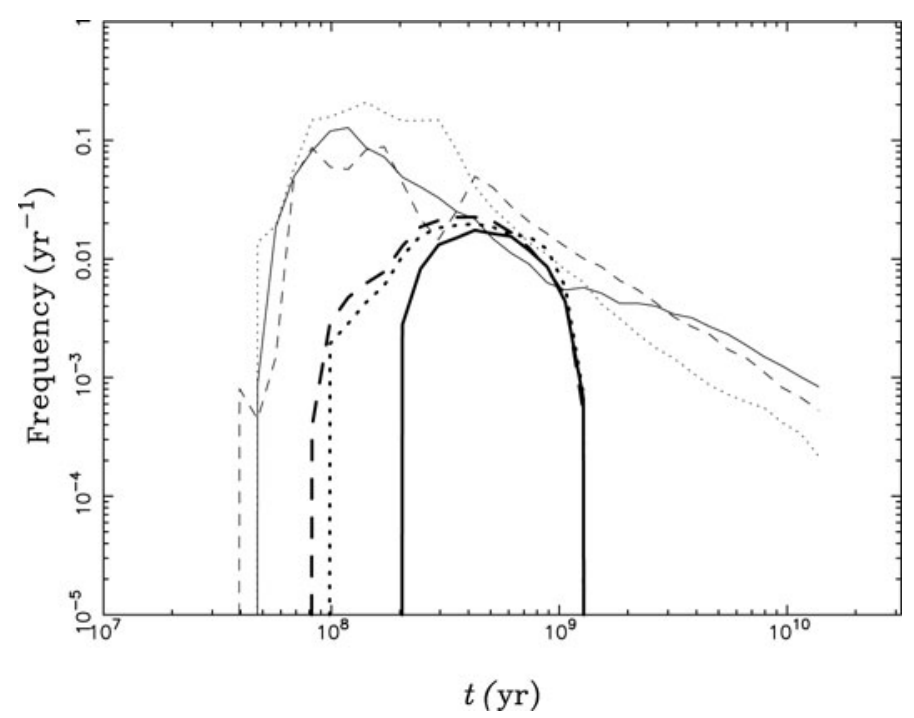

Figure 3. The evolution of birthrates of SNe Ia for a single star burst of $10^{11} M_{\odot}$ of solar metallicity. Solid, dashed and dotted lines are for $\alpha_{\mathrm{CE}}=\alpha_{\mathrm{th}}=1.0,0.75,0.5$, respectively $\left(\alpha_{\mathrm{RLOF}}=0.5\right)$. Thin lines are for the double degenerate channel, and thick lines are for the single degenerate channel.

evolve significantly with look-back time or redshift. Indeed, this is exactly what seems to have been found in recent observations (Brown et al., 2003; Rich et al., 2005).

We found that our binary model can naturally explain many observations of early-type galaxies in spite of its simplicity, and UV-upturn is expected to be universal (from dwarf to giant ellipticals; see Lisker \& Han, 2008). The model also predicts that the magnitude of UV-upturn does not depend much on metallicity or redshift. We refer the reader to Han, Podsiadlowski \& Lynas-Gray (2007) for the details.

\section{Progenitors of Type Ia supernovae}

Recent progress in cosmology is largely due to the use of Type Ia supernovae (SNe Ia) as a calibrated distance indicator (Riess et al., 1998; Perlmutter et al., 1999). The nature of their progenitors is still unclear, raising doubts as to the calibration which is purely empirical and based on nearby SN Ia sample. The SNe Ia are believed to be thermonuclear explosions of carbon-oxygen (CO) WDs. Observational characteristics of SNe Ia imply that the explosion occurs when a CO WD reaches the Chandrasekhar limit. There are mainly two channels to create Chandrasekhar-mass CO WDs: the single degenerate channel, where the CO WD accretes mass from a non-degenerate companion (Hachisu, Kato \& Nomoto, 1999a; Han \& Podsiadlowski, 2004), and the double degenerate channel, where two CO WDs with a total mass larger than the Chandrasekhar mass coalesce (Iben \& Tutukov, 1984; Webbink \& Iben, 1987) †.

Employing Eggelton's stellar evolution code (Eggleton, 1971; 1972; 1973; Han, Podsiadlowski \& Eggleton, 1994; Pols et al., 1995) and adopting the prescription of Hachisu et al. (1999) for the accretion efficiency of a CO WD, Han \& Podsiadlowski (2004) carried out detailed binary evolution calculations for about 2300 close CO WD binaries, and mapped out the initial parameters in the orbital period-secondary mass

$\dagger$ Note, however, that in this case it is quite likely that the merger product experiences core collapse rather than a thermonuclear explosion(Nomoto \& Iben, 1985). 


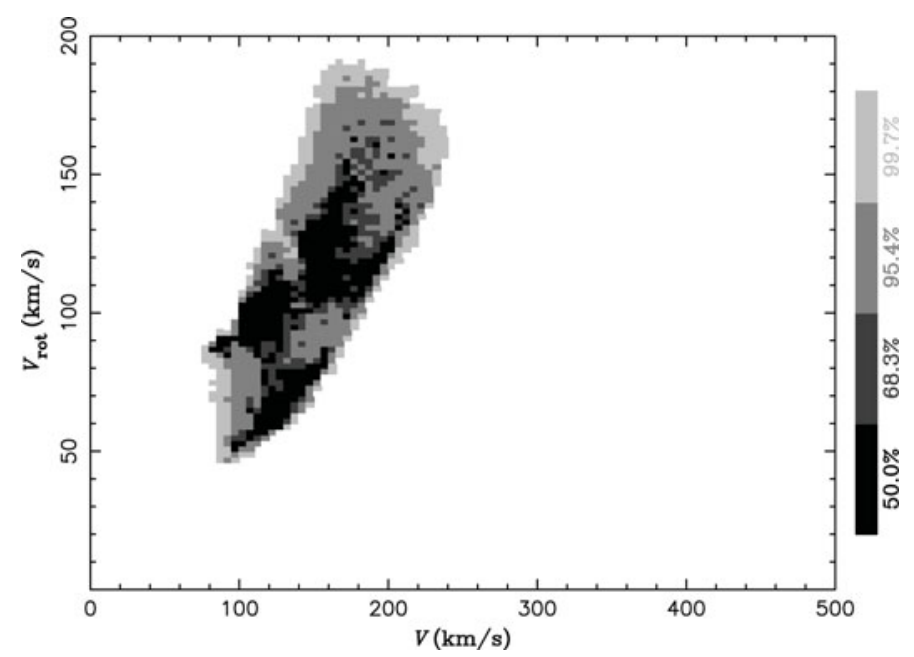

Figure 4. Snapshot probability distribution of companion stars in the plane of $\left(V_{\text {orb }}, V_{\text {rot }}\right)$ at the current epoch, where $V_{\text {orb }}$ is the orbital velocity and $V_{\text {rot }}$ the equatorial rotational velocity for the companion stars at the moment of SN explosion. The probability decreases from inner regions to outer regions. Regions, from inside to outside with corresponding gradational grey scale in the legend (from bottom to top), together with the inner regions, contain $50.0 \%, 68.3 \%$, $95.4 \%$, and $99.7 \%$ of all the systems, respectively. The model adopts a constant star formation rate over the last $15 \mathrm{Gyr}$ and $\alpha_{\mathrm{CE}}=\alpha_{\mathrm{th}}=0.75\left(\alpha_{\mathrm{RLOF}}=0.5\right)$. For a similar model but with $\alpha_{\mathrm{CE}}=\alpha_{\mathrm{th}}=1.0$, the distribution is similar, but the upper edge of $190 \mathrm{~km} / \mathrm{s}$ moves down to $170 \mathrm{~km} / \mathrm{s}$.

plane (for a range of WD masses) which lead to a SN Ia. They have implemented these results in a binary population synthesis (BPS) study to obtain the birth rates for SNe Ia for a constant star formation rate. The Galactic birth rate is lower than (but comparable to) that inferred observationally. They have also obtained the evolution of birth rates with time for a single star burst. We see from Fig. 3 that the time delay of SN Ia explosion from star burst is $\sim 0.1$ to $\sim 1$ Gyr for the single degenerate channel. The birth rates from the double degenerate channel reach to peaks at $\sim 0.1 \mathrm{Gyr}$ and decays with age (approximately $\propto t^{-1}$ ). Meng, Chen \& Han (2008) did similar investigations, but for 10 metallicities in order to investigate the metallicity effect.

For the single degenerate model, the remnant companion star after SN explosion would be a fast rotator and have a high space velocity. Fig. 4 is the distribution of companion star in the plane of orbital velocity-rotational velocity at the moment of SN explosion, as derived from a BPS study with the implementation of the results of Han \& Podsiadlowski (2004). Note, however, the ejecta of SN explosion would impact the companion and the companion obtains a kick velocity and the total velocity may be higher by up to $10 \%$ (Meng, Chen \& Han, 2007). See Han (2008) for more distributions of companion stars.

\section{Acknowledgements}

This work was in part supported by the Natural Science Foundation of China under Grant Nos 10433030, 10521001 and 2007CB815406.

\section{References}

Brown, T. M. et al. 2003, ApJ, 584, L69

Burstein, D. et al. 1988, ApJ, 328, 440

Bruzual, A. G. \& Charlot, S. 1993, ApJ, 405, 538 
Dewi, J. D. M. \& Tauris, T. M. 2000, A\&SA 360, 1043

Ferguson, H. C. et al. 1991, ApJ, 382, L69

Green, R. F., Schmidt, M., \& Liebert, J. 1986, ApJS, 61, 305

Eggleton, P. P. 1971, MNRAS, 151, 351

Eggleton, P. P. 1972, MNRAS, 156, 361

Eggleton, P. P. 1973, MNRAS, 163, 179

Hachisu, I., Kato, M., \& Nomoto, K. 1999a, ApJ, 522, 487

Hachisu, I., Kato, M., Nomoto, K., \& Umeda, H. 1999b, ApJ, 519, 314

Han Z. 1998, MNRAS, 296, 1019

Han Z. 2008a, ApJ, 677, L109

Han Z. 2008b, $A \& \mathcal{G} A$, in press (astro-ph/0804.4535)

Han, Z., Eggleton, P. P., Podsiadlowski, Ph., \& Tout, C. A. 1995b, MNRAS 277, 1443

Han Z. \& Podsiadlowski Ph. 2004, MNRAS, 350, 1301

Han, Z., Podsiadlowski, Ph., \& Eggleton, P. P. 1994, MNRAS 270, 121

Han, Z., Podsiadlowski, Ph., \& Eggleton, P. P. 1995a, MNRAS 272, 800

Han, Z., Podsiadlowski, Ph., \& Lynas-Gray, A. E. 2007, MNRAS, 380, 1098

Han, Z., Podsiadlowski, Ph., Maxted, P. F. L., Marsh, T. R., \& Ivanova, N. 2002, MNRAS, 336, 449

Han, Z., Podsiadlowski, Ph., Maxted, P. F. L., \& Marsh, T. R. 2003, MNRAS, 341, 669

Han, Z. \& Webbink, R.F. 1999, A\&A, 349, L17

Heber, U. 1986, A\& A, 155, 33

Hjellming, M. S. \& Webbink, R. F. 1987, ApJ, 318, 794

Iben, I.Jr. \& Tutukov, A. V. 1984, ApJS, 54, 335

Kilkenny, D., Koen, C., O’Donoghue, D., \& Stobie, R. S. 1997, MNRAS, 285, 640

Kurucz, R. L. 1992, in: B. Barbuy \& A. Renzini (eds.), The Stellar Populations of Galaxies, IAU Symp. (Dordrecht: Kluwer), vol. 149, p. 225

Lejeune, T., Cuisinier, F., \& Buser, R. 1997, A\& $A S, 125,229$

Lejeune, T., Cuisinier, F., \& Buser, R. 1998, A\&AS, 130, 65

Lisker, T. \& Han, Z. 2008 ApJ, in press (astro-ph/0803.2512)

Maxted, P. F. L., Heber, U., Marsh, T. R., \& North, R. C. 2001, MNRAS, 326, 1391

Meng, X., Chen, X., \& Han, Z. 2007 PASJ, 59, 835

Meng, X., Chen, X., \& Han, Z. 2008 MNRAS, submitted (astro-ph/0802.2471)

Miller, G. E. \& Scalo, J. M. 1979, ApJS 41, 513

Moni Bidin, C., Catelan, M., \& Altmann, M. 2008, A\&A, 480, L1

Moni Bidin, C., Moehler, S., Piotto, G., et al. 2006, A\&A, 451, 499

Nelemans G., Verbunt, F., Yungelson, L. R., \& Portegies Zwart, S. F. 2000, A\&A 360, 1011

Nomoto, K. \& Iben, I.Jr. 1985, ApJ, 297, 531

O'Connell, R. W. 1999, ARA\& A, 37, 603

O'Tool, S. J., Heber, U., \& Benjamin, R. A. 2004, A\&A A, 422, 1053

Paczyński, B. 1976, in: P. P. Eggleton, S. Mitton \& J. Whelan (eds.), Structure and Evolution of Close Binaries, IAU Symp. (Dordrecht: Kluwer), vol. 73, p. 75

Perlmutter, S., et al. 1999, ApJ, 517, 565

Podsiadlowski, Ph., Rappaport, S., \& Han, Z. 2003, MNRAS 341, 385

Pols, O. R., Tout, C. A., Eggleton, P. P. \& Han, Z. 1995, MNRAS, 274, 964

Rich, R. M. et al. 2005, ApJ, 619, L107

Riess, A., et al. 1998 AJ, 116, 1009

Webbink, R. F. 1988, in: J. Mikołajewska, M. Friedjung, S. J. Kenyon, \& R. Viotti (eds.), The Symbiotic Phenomenon (Dordrecht: Kluwer), p. 311

Webbink, R. F., 2007, in: EF. Milone, D. A. Leahy \& D. W. Hobill (eds.), Short Period Binary Stars, (Springer), in press (astro-ph/0704.0280)

Webbink, R. F. \& Iben, I.Jr. 1988, in: A. G. D. Philipp, D. S. Hayes \& J. W. Liebert (eds.), IAU Colloq. No. 95 (Davis Press: Schenectady), p. 445

Worthey, G. 1994, ApJS, 95, 107

Zhang, F., Li, L., \& Han, Z. 2005, MNRAS, 364, 503 


\section{Discussion}

Belczynski: Comment: Not only common envelope (CE) efficiency is very uncertain, but the very treatment of $\mathrm{CE}$ is highly uncertain. Question: How do you make single degenerate scenario of SNIa work? Since, it is known that it is rather hard to accumulate hydrogen and significantly increase white dwarf mass.

HAN: Indeed, common envelope evolution is the least understood process in binary evolution. The evolution is parameterized and calibrated with observations, which gives acceptable results. For the mass growth of white dwarf, I adopted the accumulation efficiency of Hachisu's. The mass transfer rates need to be in the right range for the WD to increase in its mass.

Christensen-Dalsgandi: Comment: Many SdB stars are observed to pulsate. Thus we may constrain their structure from observed frequencies. Question: To what extent does the structure of the SdB star depend on the formation channel? That determines an ability to distinguish them from astroseismology.

HAN: The properties of SdB stars originated from different channels are quite different. SdB stars from stable RLOF channel have thick hydrogen envelopes, the SdB stars from common envelope ejection channel have thin envelopes. The merger channel produces SdB stars with no or extremely thin hydrogen envelopes, and the mass range of the SdB stars is also larger.

VINK: You mentioned most SdBs are in binaries, which holds for the field. For clusters such as NGC 6752, Moni Bidin et al. (2006) looked for close binaries in a sample of 51 objects, but found none. Are these all mergers?

HAN: Yes. There exists a relation between SdB binary fraction and population age, for which I have submitted a Letter to A\&A. For an old stellar population, SdB stars from the merger channel dominate.

WANG: Do you care about the age of the binary components? For example if the secondary is a pre-main-sequence stars.

HAN: Both components have the same age, but the equivalent age of each component may be different. The mass gainer is rejuvenated and its equivalent age is smaller.

YI: Regarding SdB production, what is the source of the biggest uncertainty?

HAN: The biggest uncertainties are from common envelope ejection efficiency and the initial distribution of mass ratios. However, these can be calibrated b comparing theoretical results with observations of many binary related objects. We just used those calibrated parameters to produce SdB population and then applied to the UV-upturn problem in an 'a priori' way, and the UV-upturn is explained naturally. 